\title{
Correction to: Shaping Education Reform in China
}

\section{Correction to: J. Li and E. Xue, Shaping Education Reform in China, Exploring Education Policy in a Globalized World: Concepts, Contexts, and Practices, https://doi.org/10.1007/978-981-15-7745-1}

The original version of the book was inadvertently published with incorrect funding information, which has been corrected and updated as follows:

This book is funded by the research on The Key Education Thoughts of General Secretary Xi Jinping (Project No.: 18JZD006) (The Key Project of Philosophy and Social Science Research of the Ministry of Education). 